\title{
A Rapid and Cost-Effective Device for Testing Minimal Erythema Dose
}

\author{
Leah Conant · Kristen M. Beck · Wilson Liao
}

Received: July 4, 2018 / Published online: August 10, 2018

(C) The Author(s) 2018

\section{ABSTRACT}

Introduction: We describe a novel device for rapid and economical minimal erythema dose testing in patients undergoing ultraviolet (UV) light phototherapy for treatment of skin diseases.

Methods: A minimal erythema testing device was designed and created using transparent plastic sheeting and printed patterns with increasing ink density, allowing for graded UV transmission of $100 \%, 80 \%, 60 \%, 40 \%, 20 \%$, and $10 \%$ energy through six $10-\mathrm{mm}$ square apertures. The plastic sheet was placed in a UVimpenetrable and Velcro-fitted adjustable sleeve, designed to fit easily onto a patient's arm. A pilot validation study was performed, comparing this device with a commercially available windowed device in which the dose is controlled by varying the UV exposure time through sequential opening of each window. The pilot was conducted on healthy skin

Enhanced digital features To view enhanced digital features for this article go to https://doi.org/10.6084/ m9.figshare.6876212.

L. Conant

UCSF School of Medicine, University of California

San Francisco, San Francisco, CA, USA

K. M. Beck · W. Liao ( $\square)$

Department of Dermatology, University of

California San Francisco, San Francisco, CA, USA

e-mail: wilson.liao@ucsf.edu of two human subjects with different Fitzpatrick skin types.

Results: In our subjects, tested with one device on each forearm, the minimal erythema dose (MED), judged visually, was identical. However, the test device allowed MED testing in 3 min compared with $15 \mathrm{~min}$ for the traditional device. The test device is equally effective for use with ultraviolet-A (UVA), narrowband ultraviolet-B (NB-UVB) and broadband ultraviolet-B (BB-UVB) wavelengths. The test device is economical, with manufacturing cost of less than US \$2.

Conclusion: We designed an MED testing device that is quick, accurate, cost-effective, and easy to use in the setting of a busy phototherapy practice. This device therefore has many advantages over existing MED testing approaches.

Keywords: Broadband ultraviolet-B; Dermatology; MED testing; Minimal erythema dose; Narrowband ultraviolet-B; Phototesting; Phototherapy; Phototherapy device; PUVA; Ultraviolet light

\section{INTRODUCTION}

Ultraviolet (UV) light phototherapy is a wellestablished therapy for many common and uncommon dermatological ailments, including psoriasis, eczema, generalized itch, cutaneous lymphoma, and vitiligo. The minimal erythema 
dose (MED) is defined as the lowest dose of UV light that causes reddening of nondiseased skin [1]. Determining the MED is important for establishing a safe and efficacious treatment dose of UV light. Underestimating the MED may lead to below-therapeutic UV dosing and delayed clinical improvement, while overestimating the MED may lead to UV-induced burning and blistering. Moreover, even after an individual's MED is determined and phototherapy is initiated, several clinical situations may benefit from MED retesting. One example is if a patient starts a photosensitizing medication. Another example is if a patient has received phototherapy for a period of time, but has recently missed a number of treatments. While such situations can be approached using "best guess" recalibration of dosing, a quick and convenient method of accurate MED testing would be more beneficial.

\section{Existing MED Testing Methods}

A crude method used to dose UV light is based on a person's Fitzpatrick skin type, defined by a combination of degree of skin pigmentation plus patient-reported propensity to burn. In this approach, the MED is not actually measured but estimated. While this method is easy and quick, several studies have established skin typing as an inexact method of predicting skin sensitivity to light [2, 3]; For example, there are many darker-skinned individuals who require a lower starting UV dose than someone who is more fair-skinned, and patients' mental recall of their burning tendency may be imprecise.

Another approach to MED testing involves use of a UV-impenetrable template with several open windows. The template is applied to the skin, and the windows are sequentially closed during exposure to UV light, so the test area of skin is subjected to decreasing fractions of the maximum dose. Conversely, templates can be sequentially opened to expose the skin to increasing fractions of UV (e.g., DosePatch, Daavlin). In either case, after UV light exposure, the skin is assessed for redness after $24 \mathrm{~h}$ to determine the MED. The major drawback of this method is that it is time-consuming, requiring time to place the device on the patient (oftentimes requiring tape and towels to shield surrounding skin) and additional time during UV light exposure to close or open successive windows, with an interruption at each step. Another issue is that this method may also be inaccurate if the patient shifts position inside the phototherapy cabinet, causing the distance and orientation of the patient to the light source to change. For these reasons, this method may not be ideal for use in a busy, highvolume phototherapy practice.

A less commonly used method for MED testing is a handheld, semiautomated device that uses its own light source to test a range of light doses through metal filters [4-8]. A main drawback to this method is that the device is expensive, which is cost-prohibitive for many phototherapy centers. These devices cost several hundred dollars to purchase, but also require frequent maintenance. Repair or replacement of specialized parts adds to the costs of this method. Another drawback of this method is that the light source of the MED device is different from that of the phototherapy cabinet in which the patient will actually be treated, thus requiring precise calibration of both units.

UV intensity and effects are ultimately determined by many variables, including the UV light source, individual differences in skin sensitivity to light, and the skin's distance from the light source. Situational differences in these factors are the primary sources of error and confusion in the current MED testing methodology.

Currently, there is no method to perform MED testing on skin that is simultaneously convenient, accurate, rapid, and inexpensive. We describe herein an MED testing device that conveniently allows for multiple UV light doses to be tested at once. It can be manufactured at low cost, allows the user to save time in MED testing, and utilizes the phototherapy cabinet in which the patient will be treated.

\section{METHODS}

\section{Material Testing}

A variety of inexpensive UV-filtering materials were chosen and tested for effectiveness, 
filtering range, and ease of production. These included shade paint (VariShade 2, SolarSun, Inc), transparency sheets, low-density polyethylene, high-density polyethylene, polyvinylidene chloride, polypropylene, polyethylene terephthalate, and glass of varying thicknesses. In some cases, the thickness of the material required to block the appropriate proportion of UV was considered prohibitive. In other cases, the range of UV filtering was too narrow for our purposes. The authors ultimately decided to use a visually transparent, flexible, UV-filtering base of transparency sheets (VWO100C-BE, Apollo). This base was also desirable because patterns may be smoothly printed onto it to enhance its filtering capability (045 XL black laser toner cartridge 1246C001, Canon). Ultimately, an appropriately increasing gray scale was printed onto the base layer to attain the desired UV transmission for each window. To determine the relationship between the saturation of the ink printed on the transparency and the amount of UV light that was transmitted, we measured the UV transmitted through each test print of decreasing saturation (and thus increasing transparency) and recorded it as a percentage of the UV transmitted with no filter.

\section{Device Design}

The MED testing device comprised several windows on a clear plastic template (VWO100C-BE, Apollo) with laser jet ink-printed shade gradations (045 XL black laser toner cartridge 1246C001, Canon), housed in a structural support for fitting onto the arm (Fig. 1). The device allows a fixed sequence of discrete fractions of UV light doses to pass through to the underlying skin. The device is placed on the skin and exposed to UV light from the treatment source (i.e., phototherapy cabinet). A cotton sleeve with windows is used to block UV from all areas of the exposed arm except for those tested. The apertures are shaped like squares so that test results can be easily distinguished from discoloration of other common etiologies that present with erythema (bug bites, angiomas, etc.).

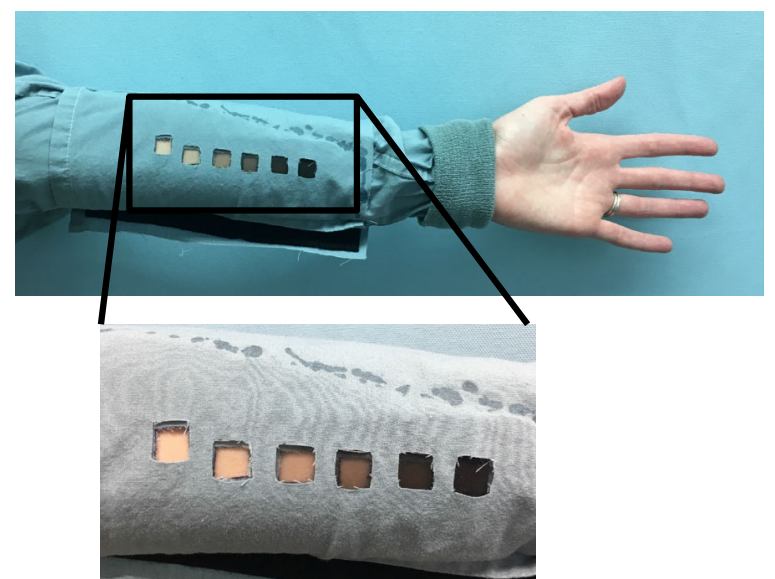

Fig. 1 Test device, comprising a cotton sleeve blocking all UV with a pocket designed to hold the transparency with printed filters of increasing absorbance. Inset shows a zoomed picture of the windows. The lightest window has no filter or transparency and allows $100 \%$ of UV through. It is offset by half a centimeter from the others to facilitate realignment with the template for reading MED $24 \mathrm{~h}$ later. The windows are shaped as squares

The phototesting template consists of printed patterns of increasing UV transmission on a transparent base with six square apertures of $10 \mathrm{~mm}$ in width, housed in a UV-impenetrable sleeve. The aperture that allows the highest UV light transmission is left open, and is offset such that the template can be easily matched to the pattern on the skin upon inspection the next day. The five remaining apertures attenuate UV radiation stepwise, resulting in relative intensities at the skin surface of $80 \%, 60 \%, 40 \%, 20 \%$, and $10 \%$ of total UV light.

\section{Measurement of UV Transmission}

UV transmission was measured in the NB-UVB, BB-UVB, and UVA spectra using light from standard clinical phototherapy bulbs (FSX72T12-UVB/HO BB-UVB bulbs by National Biological Corporation, TL100 W/01 NB-UVB bulbs by Philips, F72T12/BL/HO PUVA bulbs by Houvalite $\left.^{\circledR}\right)$. The energy transmission of UV light was measured using a UV probe meter. The amount of energy transmitted through each window of the MED device was measured serially in the phototherapy cabinet, with the UV 
probe meter positioned directly behind the window. The UV bulbs were run for $5 \mathrm{~min}$ before testing began for equilibration, and UV transmission was first measured with no filtering material to establish a maximum for comparison.

\section{Data Analysis}

Data were recorded and analyzed using Microsoft Excel and MATLAB. For each material and pattern, the filtering capability was defined as the UV measured at the probe divided by the maximum UV measured at the probe with no filtering material, expressed as a percentage. To define the relationship between the percentage transparency of the printed grayscale and percentage transmission of UV light, MATLAB's curve-fitting toolbox was used. The data were fit using both a sigmoidal fit and a linear fit for comparison. We employed the method of nonlinear least squares to find the best fit for each equation type, allowing two free fit coefficients, $a$ and $b$, for each. The linear equation was of the form

$$
y=a x+b,
$$

while the sigmoidal equation was of the form

$$
y=\frac{100}{1+\mathrm{e}^{-b(x-a)}} .
$$

For the sigmoidal function, the constant describing the upper horizontal asymptote of the function was set at 100, chosen because it is the maximum possible transmission of UV.

\section{In Vivo Validation}

The device for in vivo validation comprised a cotton sleeve with square windows of $10 \mathrm{~mm}$ in width, with the window receiving the most UV positioned off-center from the others for position verification upon inspection the next day. Beneath the windows in the sleeve was the UVfiltering material with increasing levels of UVblocking gray scale pattern. The device was positioned on the forearm of two volunteers with Fitzpatrick skin type II and III, and was exposed to up to $800 \mathrm{~mJ} / \mathrm{cm}^{2}$ of UV light in a NB-UVB phototherapy booth. The area was examined $24 \mathrm{~h}$ postexposure to determine MED. Informed consent was obtained from all participants in the study.

\section{RESULTS}

First, we wanted to determine the relationship between the saturation of the ink printed on the transparency and the amount of UV light that was transmitted. To do this, we measured the UV transmitted through each test print of decreasing saturation (and thus increasing transparency) and recorded it as a percentage of total UV. We performed this for nbUVB, bbUVB, and UVA. Theoretically, the function describing this data is sigmoidal in nature, with upper and lower horizontal asymptotes at 100\% UV transmission and $0 \%$ UV transmission accordingly. Indeed, the data were best approximated by the sigmoidal equation, with a resulting $R^{2}$ of 0.99 (Fig. 2, nbUVB shown). A linear function returned a good but less ideal fit to the data, resulting in an $R^{2}$ of 0.97 .

For in vivo testing, we housed the UV filters in a cotton sleeve (Fig. 1). The printed insert containing the filters was placed inside a specially designed pocket in the sleeve with cutouts for the testing windows. The sleeve allowed

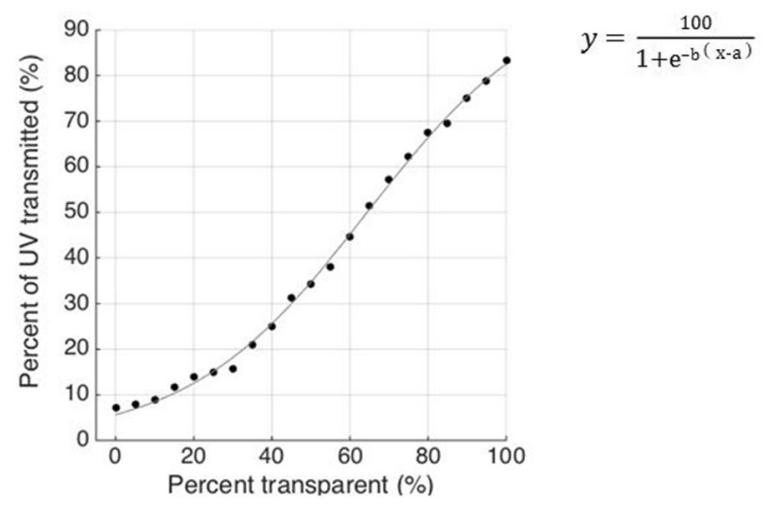

Fig. 2 Percent transmitted UV demonstrates sigmoidal relationship with percent printed transparency. Black dots represent measured UV at each tested filter as a percentage of total with no filter. Black line is the line of best fit for these data points. $R^{2}=0.998, a=64.4, b=0.044$ 
coverage of the rest of the arm while exposing only the $10 \times 10 \mathrm{~mm}$ testing sites. After $24 \mathrm{~h}$, the subjects' MED was determined as the lowest dose at which there was redness. This was compared with a windowed device (DosePatch, Daavlin) in which each window received a calculated cumulative dose of UV to correspond with the percentages of UV transmitted by the filters of the device. The MED was ascertained visually by two physicians, and was shown to be the same between the new and traditional MED devices for each subject (Fig. 3).

The overall time to complete testing with the new device was approximately $3 \mathrm{~min}$, including the time to put on the sleeve, tighten it with the Velcro fastener, slip on a glove to protect the hand, and complete testing in the phototherapy cabinet. In comparison, the overall time to complete testing with the windowed standard device was approximately $15 \mathrm{~min}$, in part due to the longer time starting and stopping delivery of UV light in the cabinet, and in part due to the longer time required to cover the exposed areas of skin around the windows. Once in the phototherapy cabinet, the time to finish testing with the device was approximately $3 \mathrm{~min}$ and $30 \mathrm{~s}$ less than with the industry-standard windowed option. The entire cost of the device is less than US $\$ 2$, and this cost could be reduced for mass production. The device is completely disposable.

\section{DISCUSSION}

\section{Performance of Device}

The novel MED device described herein performed accurate and efficient MED testing using a novel, cost-effective design. Testing of UV transmittance shows a large range of target values that may be easily allowed by the device. This range is from approximately $8 \%$ transmittance to $83 \%$ transmittance, bounded by the UV blocking capability of the ink and the baseline UV blocking of the transparency material, respectively. Because the transmittance between these bounds can be titrated by changing the saturation of print, any desired percentage of total UV is attainable. The amount of saturation needed varies approximately linearly with the transparency of the print, particularly for the transparency range $>30 \%$ (Fig. 2).

The accuracy of this method was demonstrated with two volunteers with different Fitzpatrick skin types, and it was noted that the skin had the same reaction to either receiving cumulative doses of UV to attain a total UV dose or receiving UV that was filtered to transmit decreasing fractions of energy. Both the windowed approach and the test device yielded the same MED measurement on each volunteer. With regard to efficiency, the device reduced the time in the phototherapy cabinet by approximately one-half for each subject.
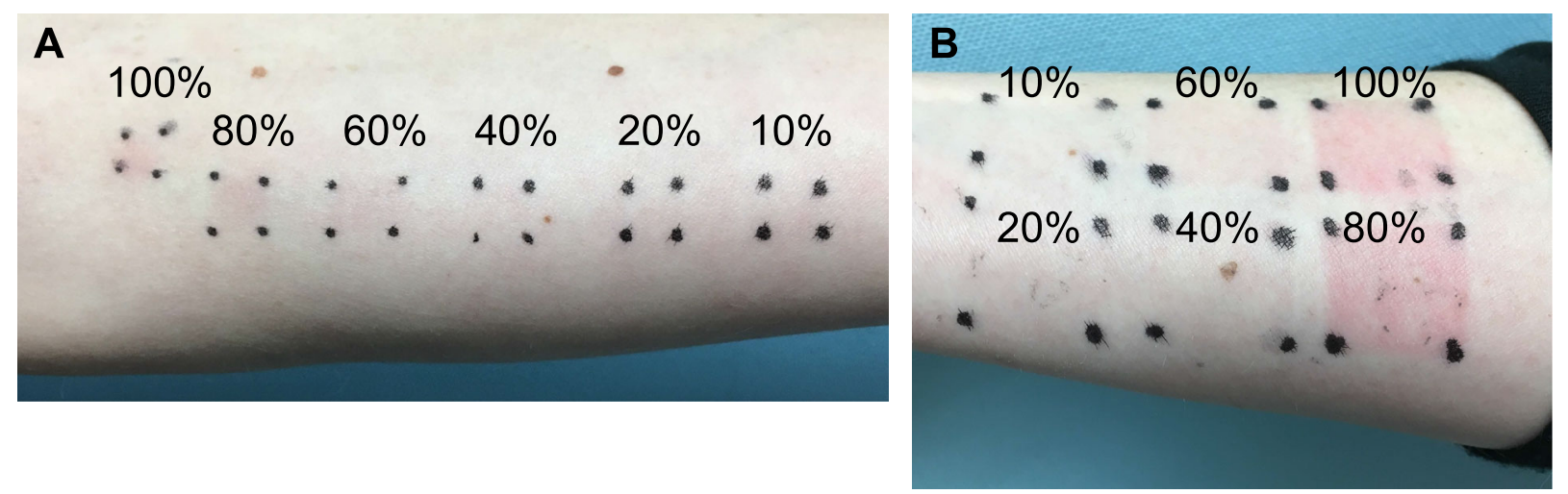

Fig. 3 MED testing results on the left and right forearms of a subject with Fitzpatrick type II. a Results with test device. b Results with windowed device. MED is visually

judged at $60 \%$ of total UV for each. Black dots mark the outline of the window through which UV passed 
Moreover, in total, the time spent using this device was one-fifth of the time spent on the peel-away standard windowed device used as a comparator.

\section{Advantages of Present Device Compared with Commercially Available MED Testing Methods}

The device described herein is advantageous because it eliminates many sources of error and confusion in the established MED testing methods. These advantages include ease of use, time efficiency, accuracy, error minimization, and economic value.

Whereas other devices may vary the exposure time and thus require multiple exposures, the device presented herein is fast and convenient to use since it requires only a single UV exposure for complete and accurate MED testing. This is because the device was designed to deliver different UV doses by varying the irradiance through each aperture of the device. This time-saving is compatible with the workflow of a high-throughput dermatology practice. Such efficiency of testing may also improve patient and provider satisfaction.

Shorter duration of testing, and fixed doses of light determined by the device itself, eliminate the human error inherent in the more time-consuming, traditional MED testing templates. With regard to the design of the device, the configuration of the windows is asymmetric, thus preventing uncertainty regarding which window is associated with which UV dose. The device is more accurate than other methods utilizing separate light sources, in part because it is designed to be used with the same light source that will be used in the patient's subsequent treatments. Because it works with the exact phototherapy bulbs that the patient will be using for future therapy, UV output mismatch between different machines is not a concern.

The device's filters work similarly to the aforementioned handheld semiautomated device, which uses expensive neutral-density filters and its own light source. However, in contrast to these semiautomated devices, this device is inexpensive; it is not part of a larger machine with its own light source. Without the need for electrical wiring, circuitry, or bulbs, the device is inexpensive and thus accessible to potential customers. Since the device is economical, it can easily be streamlined for mass production or customized for individual MED testing needs, thus increasing its cost-effectiveness and applicability.

\section{Limitations}

Although the described device has been designed to precisely filter various doses of UV light as measured by an objective UV probe meter, it has only been validated in two subjects with Fitzpatrick skin type II and III. Therefore, future studies should examine the performance of the device in a larger number of subjects with the full range of skin types.

\section{CONCLUSIONS}

The capability of this novel device to deliver several multiple UV doses at once will allow managing physicians to perform MED testing to determine optimal treatment dosing for patients efficiently and accurately. This device is an improvement over Fitzpatrick skin type estimates, because it allows the individual to be tested for skin reaction to UV light objectively. It is an improvement over cumulative UV dose testing because it does not require the practitioner to stop and restart the UV dosing every 1-2 min to adjust skin exposure. It is an improvement over handheld devices that deliver different amounts of UV light because it does not require the purchase of additional UVproducing equipment requiring maintenance. In summary, the device introduced herein is uniquely able to address the various shortcomings of the currently available MED testing methods. In doing so, it allows for increased therapeutic efficacy and reduced side effects in phototherapy. It is a rapid, economical, and accurate solution to allow for MED testing in a high-throughput, busy dermatological phototherapy practice. 


\section{ACKNOWLEDGEMENTS}

Funding. No funding or sponsorship was received for this study or publication of this article.

Authorship. All named authors meet the International Committee of Medical Journal Editors (ICMJE) criteria for authorship for this article, take responsibility for the integrity of the work as a whole, and have given their approval for this version to be published.

Disclosures. Leah Conant, Kristen Beck, and Wilson Liao have nothing to disclose.

Compliance with Ethics Guidelines. Informed consent was obtained from all participants in the study.

Open Access. This article is distributed under the terms of the Creative Commons Attribution-NonCommercial 4.0 International License (http://creativecommons.org/licenses/ by-nc/4.0/), which permits any noncommercial use, distribution, and reproduction in any medium, provided you give appropriate credit to the original author(s) and the source, provide a link to the Creative Commons license, and indicate if changes were made.

\section{REFERENCES}

1. Heckman CJ, Chandler R, Kloss JD, Benson A, Rooney D, Munshi T, et al. Minimal Erythema Dose (MED) testing. J Vis Exp JoVE. 2013;75:e50175. https://doi. org/10.3791/50175.
2. Stern RS, Momtaz K. Skin typing for assessment of skin cancer risk and acute response to UV-B and oral methoxsalen photochemotherapy. Arch Dermatol. 1984;120(7):869-73.

3. Sayre RM, Desrochers DL, Wilson CJ, Marlowe E. Skin type, minimal erythema dose (MED), and sunlight acclimatization. J Am Acad Dermatol. 1981;5(4):439-43.

4. Otman SG, Edwards C, Gambles B, Anstey AV. Validation of a semiautomated method of minimal erythema dose testing for narrowband ultraviolet $\mathrm{B}$ phototherapy. Br J Dermatol. 2006;155(2):416-21. https://doi.org/10.1111/j.1365-2133.2006.07273.x.

5. Wishart J. A new method of phototesting before narrowband UVB therapy. Photodermatol Photoimmunol Photomed. 2001;17(4):197-9.

6. Morita A, Shintani Y, Nishida E, Kato H, Yoshida H, Minamoto $\mathrm{M}$, et al. Feasibility and accuracy of a newly developed hand-held device with a flat-type fluorescent lamp for measuring the minimal erythema dose for narrow-band UVB therapy. Photodermatol Photoimmunol Photomed. 2009;25(1):41-4. https://doi.org/10.1111/j.1600-0781.2009.00402.x.

7. Diffey BL, De Berker DA, Saunders PJ, Farr PM. A device for phototesting patients before PUVA therapy. Br J Dermatol. 1993;129(6):700-3.

8. Gordon PM, Saunders PJ, Diffey BL, Farr PM. Phototesting prior to narrowband (TL-01) ultraviolet B phototherapy. Br J Dermatol. 1998;139(5):811-4. 